\title{
A Class of Exponential Ratio Estimators of Finite Population Mean Using Two Auxiliary Variables
}

\author{
Prayas Sharma \\ Department of QT \& RM \\ University of Petroleum and Energy Studies, Dehradun-248001 \\ prayassharma02@gmail.com \\ Rajesh Singh \\ Department of Statistics \\ Banaras Hindu University, Varanasi-221005 \\ rsinghstat@gmail.com
}

\begin{abstract}
This paper proposes a class of estimators based on information of two auxiliary variables. The expressions of mean square errors of the proposed class of estimators are derived in a general form. It is shown that the proposed class of estimators is always more efficient than regression estimator based on two variable, estimators proposed by Abu-Dayeh (2003) and estimators recently proposed by and Lu and Yan (2014). In addition, we support this theoretical result by an empirical study using original data to show the superiority of the constructed estimators over others.
\end{abstract}

Keywords: Auxiliary variable, Mean square error, Simple random sampling.

\section{Introduction}

In the theory of sample surveys, it is usual to make use of the auxiliary information at the estimation stage in order to improve the precision of an estimator of unknown population parameter of interest. Ratio, product and regression methods of estimation are good examples in this context. Several authors including Hartley-Ross (1954), Quenouille's (1956), Khoshnevisan et al. (2007), Singh et al. (2007), and Singh and Kumar (2011), Verma et al. (2015) have considered the problem of estimating the mean of a survey variable when auxiliary variables are made available. In most of the cases, we see that instead of one auxiliary variable, information of two auxiliary variables are available. When two auxiliary variables are present Singh (1965, 1967), Perri (2007), Abu-Dayeh et al. (2003), Kadilar and Cingi (2005), Tailor et al. (2012), Singh and Kumar (2012), Lu (2013), Sharma and Singh (2014), and Lu and Yan (2014) suggested some estimators for estimating the population mean. For recent development, exponential estimators have been widely studied by several authors including Lu et al. (2014) and Koyuncu and Ozel (2013) to minimize the mean square errors of estimators under different situations.

Let $\mathrm{U}=\left(\mathrm{U}_{1}, \mathrm{U}_{2}, \mathrm{U}_{3}, \ldots ., \mathrm{U}_{\mathrm{i}}, \ldots, \mathrm{U}_{\mathrm{N}}\right)$ denotes a finite population of distinct and identifiable units. For estimating the population mean $\bar{Y}$ of a study variable Y, let us consider $X_{1}$ and $X_{2}$ are the two auxiliary variables that are correlated with study variable $\mathrm{Y}$, taking the corresponding values of the units. Let a sample of size $\mathrm{n}$ be drawn from this population using simple random sampling without replacement (SRSWOR) and 
$\bar{y}, \bar{x}_{1}$ and $\bar{x}_{2}$ respectively denote the sample means of the variables $y, x_{1}$ and $x_{2}$. We assume that population means $\overline{\mathrm{X}}_{1}$ and $\overline{\mathrm{X}}_{2}$ of the auxiliary variables are known.

Let us define,

$$
\mathrm{e}_{0}=\frac{(\overline{\mathrm{y}}-\overline{\mathrm{Y}})}{\overline{\mathrm{Y}}}, \quad \mathrm{e}_{1}=\frac{\left(\overline{\mathrm{x}}_{1}-\overline{\mathrm{X}}_{1}\right)}{\overline{\mathrm{X}}_{1}}, \quad \mathrm{e}_{2}=\frac{\left(\overline{\mathrm{x}}_{2}-\overline{\mathrm{X}}_{2}\right)}{\overline{\mathrm{X}}_{2}}
$$

Such that,

$$
\begin{aligned}
& E\left(e_{i}\right)=0,(i=0,1,2), E\left(e_{0}^{2}\right)=f_{1} C_{y}^{2}, E\left(e_{1}^{2}\right)=f_{1} C_{x_{1}}^{2}, E\left(e_{2}^{2}\right)=f_{1} C_{x_{2}}^{2}, E\left(e_{1} e_{0}\right)=f_{1} \rho_{y_{1}} C_{y} C_{x_{1}} \\
& E\left(e_{2} e_{0}\right)=f_{1} \rho_{y_{x_{2}}} C_{y} C_{x_{2}}, \quad E\left(e_{1} e_{2}\right)=f_{1} \rho_{x_{1} x_{2}} C_{x_{1}} C_{x_{2}}, \text { where, } f_{1}=\left(\frac{1}{n}-\frac{1}{N}\right), C_{y}^{2}=\frac{S_{y}^{2}}{\bar{Y}^{2}}, \\
& C_{x}^{2}=\frac{S_{x}^{2}}{\bar{X}^{2}}, \quad S_{y}^{2}=(N-1)^{-1} \sum_{i=1}^{N}\left(Y_{i}-\bar{Y}\right)^{2}, S_{x}^{2}=(N-1)^{-1} \sum_{i=1}^{N}\left(X_{i}-\bar{X}\right)^{2}, \quad \rho_{y x}=\frac{S_{y x}}{\sqrt{S_{y}^{2} S_{x}^{2}}},
\end{aligned}
$$

Also, $\rho_{\mathrm{yx}_{1}}, \rho_{\mathrm{yx}_{2}}$ and $\rho_{\mathrm{x}_{1} \mathrm{x}_{2}}$ denote the correlation coefficients between $\mathrm{Y}$ and $\mathrm{X}_{1}, \mathrm{Y}$ and $\mathrm{X}_{2}$ and $\mathrm{X}_{1}$ and $\mathrm{X}_{2}$ respectively and $\mathrm{C}_{\mathrm{y}}, \mathrm{C}_{\mathrm{x}_{1}}$ and $\mathrm{C}_{\mathrm{x}_{2}}$ denote the coefficients of variation of $\mathrm{Y}$ on $\mathrm{X}_{1}$ and $\mathrm{X}_{2}$ respectively.

The traditional multivariate ratio estimator using information of two auxiliary variables to estimate the population mean $\bar{Y}$, as follows

$$
\mathrm{t}_{\mathrm{MR}}=\theta_{1} \overline{\mathrm{y}} \frac{\overline{\mathrm{X}}_{1}}{\overline{\mathrm{x}}_{1}}+\theta_{2} \overline{\mathrm{y}} \frac{\overline{\mathrm{X}}_{2}}{\overline{\mathrm{x}}_{2}}
$$

where $\bar{x}_{i}$ and $\bar{X}_{i}(i=1,2)$ denote respectively the sample and the population means of the variable $\bar{x}_{i} \cdot \theta_{1}$ and $\theta_{1}$ are the weights that satisfy the condition: $\theta_{1}+\theta_{2}=1$

The minimum MSE of this estimator is given by

$\operatorname{MSE}_{\min }\left(\mathrm{t}_{\mathrm{MR}}\right)=\mathrm{f}_{1} \overline{\mathrm{Y}}^{2}\left(\mathrm{C}_{\mathrm{y}}^{2}+\theta_{1}^{2} \mathrm{C}_{\mathrm{x}_{1}}^{2}+\theta_{2}^{2} \mathrm{C}_{\mathrm{x}_{2}}^{2}-2 \theta_{1} \rho_{\mathrm{yx}_{1}} \mathrm{C}_{\mathrm{y}} \mathrm{C}_{\mathrm{x}_{1}}-2 \theta_{2} \rho_{\mathrm{yx}_{2}} \mathrm{C}_{\mathrm{y}} \mathrm{C}_{\mathrm{x}_{2}}+2 \theta_{1} \theta_{2} \rho_{\mathrm{x}_{1} \mathrm{x}_{2}} \mathrm{C}_{\mathrm{x}_{1}} \mathrm{C}_{\mathrm{x}_{2}}\right)$

where $\theta_{1}=\frac{C_{x_{2}}^{2}-\rho_{y_{2}} C_{y} C_{x_{2}}+\rho_{y_{1}} C_{y} C_{x_{1}}-\rho_{x_{1} x_{2}} C_{x_{1}} C_{x_{2}}}{C_{x_{1}}^{2}+C_{x_{2}}^{2}-2 \rho x_{1} x_{2} C_{x_{1}} C_{x_{2}}}$ and $\theta_{2}=1-\theta_{1}$

Abu-Dayeh et al. (2003) proposed the following two estimators using two auxiliary variables

$$
\mathrm{t}_{\mathrm{r} 1}=\overline{\mathrm{y}}\left(\frac{\overline{\mathrm{x}}_{1}}{\overline{\mathrm{X}}_{1}}\right)^{\gamma_{1}}\left(\frac{\overline{\mathrm{x}}_{2}}{\overline{\mathrm{X}}_{2}}\right)^{\gamma_{2}}
$$




$$
\mathrm{t}_{\mathrm{r} 2}=\lambda_{1} \overline{\mathrm{y}}\left(\frac{\overline{\mathrm{x}}_{1}}{\overline{\mathrm{X}}_{1}}\right)^{\gamma_{1}}+\lambda_{2} \overline{\mathrm{y}}\left(\frac{\overline{\mathrm{x}}_{2}}{\overline{\mathrm{X}}_{2}}\right)^{\gamma_{2}}
$$

where $\gamma_{1}, \gamma_{2}, \lambda_{1}$ and $\lambda_{2}\left(\lambda_{1}+\lambda_{2}=1\right)$ are suitably chosen constant for minimizing the mean square errors of the estimators $t_{r 1}$ and $t_{r 2}$.

The minimum MSE expressions of the estimators $t_{\mathrm{r} 1}$ and $t_{\mathrm{r} 2}$ are respectively given as

$$
\operatorname{MSE}_{\min }\left(\mathrm{t}_{\mathrm{r} 1}\right)=\mathrm{f}_{1} \overline{\mathrm{Y}}^{2} \mathrm{C}_{\mathrm{y}}^{2}\left(1-\frac{\rho_{\mathrm{yx}_{1}}^{2}+\rho_{\mathrm{yx}_{2}}^{2}-2 \rho_{\mathrm{yx}_{1}} \rho_{\mathrm{yx}_{2}} \rho_{\mathrm{x}_{1} \mathrm{x}_{2}}}{1-\rho_{\mathrm{x}_{1} \mathrm{x}_{2}}^{2}}\right)
$$

$$
\begin{aligned}
\operatorname{MSE}_{\min } & \left(\mathrm{t}_{\mathrm{r} 2}\right)=\mathrm{f}_{1} \overline{\mathrm{Y}}^{2}\left(\mathrm{C}_{\mathrm{y}}^{2}+\lambda_{1}^{2} \gamma_{1}^{2} \mathrm{C}_{\mathrm{x}_{1}}^{2}+\lambda_{2}^{2} \gamma_{2}^{2} \mathrm{C}_{\mathrm{x}_{2}}^{2}-2 \gamma_{1} \lambda_{1} \rho_{\mathrm{yx}_{1}} \mathrm{C}_{\mathrm{y}} \mathrm{C}_{\mathrm{x}_{1}}-2 \lambda_{2} \gamma_{1} \rho_{\mathrm{yx}_{2}} \mathrm{C}_{\mathrm{y}} \mathrm{C}_{\mathrm{x}_{2}}\right. \\
& \left.+2 \lambda_{1} \lambda_{2} \gamma_{1} \gamma_{2} \rho_{\mathrm{x}_{1} \mathrm{x}_{2}} \mathrm{C}_{\mathrm{x}_{1}} \mathrm{C}_{\mathrm{x}_{2}}\right)
\end{aligned}
$$

where $\lambda_{1}=\frac{\gamma_{2}^{2} \mathrm{C}_{\mathrm{x}_{2}}^{2}-\gamma_{1} \rho_{\mathrm{yx}_{2}} \mathrm{C}_{\mathrm{y}} \mathrm{C}_{\mathrm{x}_{2}}+\gamma_{2} \rho_{\mathrm{yx}_{1}} \mathrm{C}_{\mathrm{y}} \mathrm{C}_{\mathrm{x}_{1}}-\gamma_{1} \gamma_{2} \rho_{\mathrm{x}_{1} \mathrm{x}_{2}} \mathrm{C}_{\mathrm{x}_{1}} \mathrm{C}_{\mathrm{x}_{2}}}{\gamma_{1}^{2} \mathrm{C}_{\mathrm{x}_{1}}^{2}+\gamma_{2}^{2} \mathrm{C}_{\mathrm{x}_{2}}^{2}-2 \gamma_{1} \gamma_{2} \rho_{\mathrm{x}_{1} \mathrm{x}_{2}} \mathrm{C}_{\mathrm{x}_{1}} \mathrm{C}_{\mathrm{x}_{2}}}$ and $\lambda_{2}=1-\lambda_{1}$

when the information is available on two auxiliary variables the well known regression estimator is given as

$$
\mathrm{t}_{\mathrm{reg}}=\overline{\mathrm{y}}+\mathrm{b}_{1}\left(\overline{\mathrm{X}}_{1}-\overline{\mathrm{x}}_{1}\right)+\mathrm{b}_{2}\left(\overline{\mathrm{X}}_{2}-\overline{\mathrm{x}}_{2}\right)
$$

where $\mathrm{b}_{1}=\frac{\mathrm{s}_{\mathrm{yx}_{1}}}{\mathrm{~s}_{\mathrm{x}_{1}}^{2}}$ and $\mathrm{b}_{2}=\frac{\mathrm{s}_{\mathrm{yx}_{2}}}{\mathrm{~s}_{\mathrm{x}_{2}}^{2}}$

The minimum MSE of the estimator $t_{\text {reg }}$ is given by,

$$
\operatorname{MSE}_{\text {min }}\left(\mathrm{t}_{\mathrm{reg}}\right)=\mathrm{f}_{1} \mathrm{~S}_{\mathrm{y}}^{2}\left(1-\rho_{\mathrm{yx}_{1}}^{2}-\rho_{\mathrm{yx}_{2}}^{2}+2 \rho_{\mathrm{yx}_{1}} \rho_{\mathrm{yx}_{2}} \rho_{\mathrm{x}_{1} \mathrm{x}_{2}}\right)
$$

Lu and Yan (2014) proposed a class of multivariate ratio estimator using information of two auxiliary variables given by

$$
\mathrm{t}_{\mathrm{L}}=\mathrm{k}_{1} \overline{\mathrm{y}} \frac{\mathrm{a}_{1} \overline{\mathrm{X}}_{1}+\mathrm{b}_{1}}{\mathrm{a}_{1} \overline{\mathrm{x}}_{1}+\mathrm{b}_{1}}+\mathrm{k}_{2} \overline{\mathrm{y}} \frac{\mathrm{a}_{2} \overline{\mathrm{X}}_{2}+\mathrm{b}_{2}}{\mathrm{a}_{2} \overline{\mathrm{x}}_{2}+\mathrm{b}_{2}}
$$

where $\mathrm{k}_{1}$ and $\mathrm{k}_{2}$ are suitable constant in order to minimize the mean square error of estimator $\mathrm{t}_{\mathrm{L}}$ and $\mathrm{k}_{1}+\mathrm{k}_{2}=1, \mathrm{a}_{1} \neq 0, \mathrm{a}_{2} \neq 0, \mathrm{~b}_{1}$ and $\mathrm{b}_{2}$ are either real numbers or functions of known parameters.

The minimum MSE of this class of estimators is given by

$$
\begin{gathered}
\operatorname{MSE}_{\min }\left(\mathrm{t}_{\mathrm{L}}\right)=\mathrm{f}_{1} \overline{\mathrm{Y}}^{2}\left(\mathrm{C}_{\mathrm{y}}^{2}+\mathrm{k}_{1}^{2} \alpha^{2} \mathrm{C}_{\mathrm{x}_{1}}^{2}+\mathrm{k}_{2}^{2} \alpha^{2} \mathrm{C}_{\mathrm{x}_{2}}^{2}-2 \mathrm{k}_{1} \alpha_{1} \rho_{\mathrm{yx}_{1}} \mathrm{C}_{\mathrm{y}} \mathrm{C}_{\mathrm{x}_{1}}-2 \mathrm{k}_{2} \alpha_{2} \rho_{\mathrm{yx}_{2}} \mathrm{C}_{\mathrm{y}} \mathrm{C}_{\mathrm{x}_{2}}\right. \\
\left.+2 \mathrm{k}_{1} \mathrm{k}_{2} \alpha_{1} \alpha_{2} \rho_{\mathrm{x}_{1} \mathrm{x}_{2}} \mathrm{C}_{\mathrm{x}_{1}} \mathrm{C}_{\mathrm{x}_{2}}\right)
\end{gathered}
$$




\section{The Proposed class of estimators}

We propose an improved class of estimators for estimating $\bar{Y}$ when information of two auxiliary variables is available, as

$$
\mathrm{t}_{\mathrm{N}}=\overline{\mathrm{y}}\left[\mathrm{w}_{1}\left(\frac{\overline{\mathrm{x}}_{1}}{\overline{\mathrm{X}}_{1}}\right)^{\delta} \exp \left\{\frac{\eta_{1}\left(\overline{\mathrm{x}}_{1}-\overline{\mathrm{x}}_{1}\right)}{\eta_{1}\left(\overline{\mathrm{x}}_{1}+\overline{\mathrm{x}}_{1}\right)+2 \lambda_{1}}\right\}+\mathrm{w}_{2}\left(\frac{\overline{\mathrm{x}}_{2}}{\overline{\mathrm{x}}_{2}}\right)^{\beta} \exp \left\{\frac{\eta_{2}\left(\overline{\mathrm{x}}_{2}-\overline{\mathrm{x}}_{2}\right)}{\eta_{2}\left(\overline{\mathrm{x}}_{2}+\overline{\mathrm{x}}_{2}\right)+2 \lambda_{2}}\right\}\right]
$$

where $\delta$ and $\beta$ are constants that can takes values $(0,1,-1)$ for designing different estimators; $\eta_{1}, \lambda_{1}, \eta_{2}$, and $\lambda_{2}$ are either real numbers or the function of the known parameters. $\mathrm{w}_{1}$ and $\mathrm{w}_{2}$ are suitable chosen constants to be determined such that mean square error (MSE) of the class of estimators $t_{N}$ is minimum.

It is to be mentioned that

(i) For $\left(\mathrm{w}_{1}, \mathrm{w}_{2}\right)=(1,0)$, the class of estimator $\mathrm{t}_{\mathrm{N}}$ reduces to the class of estimator as

$$
\mathrm{t}_{\mathrm{Nk}}=\overline{\mathrm{y}}\left\{\mathrm{w}_{1}\left(\frac{\overline{\mathrm{x}}_{1}}{\overline{\mathrm{X}}_{1}}\right)^{\delta} \exp \left(\frac{\eta_{1}\left(\overline{\mathrm{X}}_{1}-\overline{\mathrm{x}}_{1}\right)}{\eta_{1}\left(\overline{\mathrm{X}}_{1}+\overline{\mathrm{x}}_{1}\right)+2 \lambda_{1}}\right)\right\}
$$

A set of new estimators generated from (2.1) using suitable values of $\delta, \beta, \eta_{1}, \eta_{2}, \lambda_{1}$ and $\lambda_{2}$ are listed below

$$
\begin{aligned}
& \mathrm{t}_{\mathrm{N} 1}=\overline{\mathrm{y}}\left[\mathrm{w}_{1} \exp \left\{\frac{\left(\overline{\mathrm{X}}_{1}-\overline{\mathrm{x}}_{1}\right)}{\left(\mathrm{X}_{1}+\mathrm{x}_{1}\right)+2}\right\}+\mathrm{w}_{2} \exp \left\{\frac{\left(\overline{\mathrm{X}}_{2}-\overline{\mathrm{x}}_{2}\right)}{\left(\overline{\mathrm{X}}_{2}+\overline{\mathrm{x}}_{2}\right)+2}\right\}\right] \\
& \mathrm{t}_{\mathrm{N} 2}=\overline{\mathrm{y}}\left[\mathrm{w}_{1}\left(\frac{\overline{\mathrm{X}}_{1}}{\overline{\mathrm{x}}_{1}}\right) \exp \left\{\frac{\left(\overline{\mathrm{X}}_{1}-\overline{\mathrm{x}}_{1}\right)}{\left(\overline{\mathrm{X}}_{1}+\overline{\mathrm{x}}_{1}\right)+2}\right\}+\mathrm{w}_{2}\left(\frac{\overline{\mathrm{X}}_{2}}{\overline{\mathrm{x}}_{2}}\right) \exp \left\{\frac{\left(\overline{\mathrm{X}}_{2}-\overline{\mathrm{x}}_{2}\right)}{\left(\overline{\mathrm{X}}_{2}+\overline{\mathrm{x}}_{2}\right)+2}\right\}\right] \\
& \mathrm{t}_{\mathrm{N} 3}=\overline{\mathrm{y}}\left[\mathrm{w}_{1}\left(\frac{\overline{\mathrm{X}}_{1}}{\overline{\mathrm{x}}_{1}}\right) \exp \left\{\frac{\mathrm{Cx}_{1}\left(\overline{\mathrm{X}}_{1}-\overline{\mathrm{x}}_{1}\right)}{\mathrm{Cx}_{1}\left(\overline{\mathrm{X}}_{1}+\overline{\mathrm{x}}_{1}\right)+2}\right\}+\mathrm{w}_{2}\left(\frac{\overline{\mathrm{X}}_{2}}{\overline{\mathrm{x}}_{2}}\right) \exp \left\{\frac{\left(\overline{\mathrm{X}}_{2}-\overline{\mathrm{x}}_{2}\right)}{\left(\overline{\mathrm{X}}_{2}+\overline{\mathrm{x}}_{2}\right)+2}\right\}\right] \\
& \mathrm{t}_{\mathrm{N} 4}=\overline{\mathrm{y}}\left[\mathrm{w}_{1}\left(\frac{\overline{\mathrm{X}}_{1}}{\overline{\mathrm{x}}_{1}}\right) \exp \left\{\frac{\beta_{2}\left(\mathrm{x}_{1}\right)\left(\overline{\mathrm{X}}_{1}-\overline{\mathrm{x}}_{1}\right)}{\beta_{2}\left(\mathrm{x}_{1}\right)\left(\overline{\mathrm{X}}_{1}+\overline{\mathrm{x}}_{1}\right)+2}\right\}+\mathrm{w}_{2}\left(\frac{\overline{\mathrm{X}}_{2}}{\overline{\mathrm{x}}_{2}}\right) \exp \left\{\frac{\left(\overline{\mathrm{X}}_{2}-\overline{\mathrm{x}}_{2}\right)}{\left(\overline{\mathrm{X}}_{2}+\overline{\mathrm{x}}_{2}\right)+2}\right\}\right] \\
& \mathrm{t}_{\mathrm{N} 5}=\overline{\mathrm{y}}\left[\mathrm{w}_{1}\left(\frac{\overline{\mathrm{X}}_{1}}{\overline{\mathrm{x}}_{1}}\right) \exp \left\{\frac{\mathrm{C}_{\mathrm{x}_{1}}\left(\overline{\mathrm{X}}_{1}-\overline{\mathrm{x}}_{1}\right)}{\mathrm{C}_{\mathrm{x}_{1}}\left(\overline{\mathrm{X}}_{1}+\overline{\mathrm{x}}_{1}\right)+2}\right\}+\mathrm{w}_{2}\left(\frac{\overline{\mathrm{X}}_{2}}{\overline{\mathrm{x}}_{2}}\right) \exp \left\{\frac{\mathrm{C}_{\mathrm{x}_{2}}\left(\overline{\mathrm{X}}_{2}-\overline{\mathrm{x}}_{2}\right)}{\mathrm{C}_{\mathrm{x}_{2}}\left(\overline{\mathrm{X}}_{2}+\overline{\mathrm{x}}_{2}\right)+2}\right\}\right] \\
& \mathrm{t}_{\mathrm{N} 6}=\overline{\mathrm{y}}\left[\mathrm{w}_{1}\left(\frac{\overline{\mathrm{X}}_{1}}{\overline{\mathrm{x}}_{1}}\right) \exp \left\{\frac{\beta_{2}\left(\mathrm{x}_{1}\right)\left(\overline{\mathrm{X}}_{1}-\overline{\mathrm{x}}_{1}\right)}{\left(\overline{\mathrm{X}}_{1}+\overline{\mathrm{x}}_{1}\right)+2}\right\}+\mathrm{w}_{2}\left(\frac{\overline{\mathrm{X}}_{2}}{\overline{\mathrm{x}}_{2}}\right) \exp \left\{\frac{\beta_{2}\left(\mathrm{x}_{2}\right)\left(\overline{\mathrm{X}}_{2}-\overline{\mathrm{x}}_{2}\right)}{\left(\overline{\mathrm{X}}_{2}+\overline{\mathrm{x}}_{2}\right)+2}\right\}\right] \\
& \mathrm{t}_{\mathrm{N} 7}=\overline{\mathrm{y}}\left[\mathrm{w}_{1}\left(\frac{\overline{\mathrm{X}}_{1}}{\overline{\mathrm{x}}_{1}}\right) \exp \left\{\frac{\beta_{1}\left(\mathrm{x}_{1}\right)\left(\overline{\mathrm{x}}_{1}-\overline{\mathrm{x}}_{1}\right)}{\beta_{1}\left(\mathrm{x}_{1}\right)\left(\overline{\mathrm{X}}_{1}+\overline{\mathrm{x}}_{1}\right)+2}\right\}+\mathrm{w}_{2}\left(\frac{\overline{\mathrm{X}}_{2}}{\overline{\mathrm{x}}_{2}}\right) \exp \left\{\frac{\beta_{2}\left(\mathrm{x}_{2}\right)\left(\overline{\mathrm{X}}_{2}-\overline{\mathrm{x}}_{2}\right)}{\beta_{2}\left(\mathrm{x}_{2}\right)\left(\overline{\mathrm{X}}_{2}+\overline{\mathrm{x}}_{2}\right)+2}\right\}\right]
\end{aligned}
$$




$$
\begin{aligned}
& \mathrm{t}_{\mathrm{N} 8}=\overline{\mathrm{y}}\left[\mathrm{w}_{1}\left(\frac{\overline{\mathrm{X}}_{1}}{\overline{\mathrm{x}}_{1}}\right) \exp \left\{\frac{\beta_{2}\left(\mathrm{x}_{1}\right)\left(\overline{\mathrm{x}}_{1}-\overline{\mathrm{x}}_{1}\right)}{\beta_{2}\left(\mathrm{x}_{1}\right)\left(\overline{\mathrm{x}}_{1}+\overline{\mathrm{x}}_{1}\right)+2 \mathrm{Cx}_{1}}\right\}+\mathrm{w}_{2}\left(\frac{\mathrm{x}_{2}}{\overline{\mathrm{x}}_{2}}\right) \exp \left\{\frac{\beta_{2}\left(\mathrm{x}_{2}\right)\left(\overline{\mathrm{x}}_{2}-\overline{\mathrm{x}}_{2}\right)}{\beta_{2}\left(\mathrm{x}_{2}\right)\left(\overline{\mathrm{x}}_{2}+\overline{\mathrm{x}}_{2}\right)+2 \mathrm{Cx} \mathrm{x}_{2}}\right\}\right] \\
& \mathrm{t}_{\mathrm{N} 9}=\overline{\mathrm{y}}\left[\mathrm{w}_{1}\left(\frac{\overline{\mathrm{X}}_{1}}{\overline{\mathrm{x}}_{1}}\right) \exp \left\{\frac{\beta_{2}\left(\mathrm{x}_{1}\right)\left(\overline{\mathrm{X}}_{1}-\overline{\mathrm{x}}_{1}\right)}{\beta_{2}\left(\mathrm{x}_{1}\right)\left(\overline{\mathrm{x}}_{1}+\overline{\mathrm{x}}_{1}\right)}\right\}+\mathrm{w}_{2}\left(\frac{\overline{\mathrm{X}}_{2}}{\overline{\mathrm{x}}_{2}}\right) \exp \left\{\frac{\beta_{2}\left(\mathrm{x}_{2}\right)\left(\overline{\mathrm{X}}_{2}-\overline{\mathrm{x}}_{2}\right)}{\left.\left.\beta_{2}\left(\mathrm{x}_{2}\right)\left(\overline{\mathrm{x}}_{2}+\overline{\mathrm{x}}_{2}\right)\right\}\right]}\right]\right.
\end{aligned}
$$

Table 2.1: Suitable choices of $\delta, \beta, \eta_{1}, \eta_{2}, \lambda_{1}$ and $\lambda_{2}$

\begin{tabular}{ccccccc}
\hline Estimators & $\delta$ & $\eta_{1}$ & $\lambda_{1}$ & $\beta$ & $\eta_{2}$ & $\lambda_{2}$ \\
\hline $\mathrm{t}_{\mathrm{N} 1}$ & 0 & 1 & 1 & 0 & 1 & 1 \\
$\mathrm{t}_{\mathrm{N} 2}$ & -1 & 1 & 1 & -1 & 1 & 1 \\
$\mathrm{t}_{\mathrm{N} 3}$ & -1 & $\mathrm{Cx}$ & 1 & -1 & 1 & 1 \\
$\mathrm{t}_{\mathrm{N} 4}$ & -1 & $\beta_{2}\left(\mathrm{x}_{1}\right)$ & 1 & -1 & 1 & 1 \\
$\mathrm{t}_{\mathrm{N} 5}$ & -1 & $\mathrm{Cx}_{1}$ & 1 & -1 & $\mathrm{Cx}_{2}$ & 1 \\
$\mathrm{t}_{\mathrm{N} 6}$ & -1 & $\beta_{2}\left(\mathrm{x}_{1}\right)$ & 1 & -1 & $\beta_{2}\left(\mathrm{x}_{2}\right)$ & 1 \\
$\mathrm{t}_{\mathrm{N} 7}$ & -1 & $\beta_{2}\left(\mathrm{x}_{1}\right)$ & 1 & -1 & $\beta_{2}\left(\mathrm{x}_{2}\right)$ & 1 \\
$\mathrm{t}_{\mathrm{N} 8}$ & -1 & $\beta_{2}\left(\mathrm{x}_{1}\right)$ & $\mathrm{Cx}$ & -1 & $\beta_{2}\left(\mathrm{x}_{2}\right)$ & $\mathrm{Cx}$ \\
$\mathrm{t}_{\mathrm{N} 9}$ & -1 & $\beta_{2}\left(\mathrm{x}_{1}\right)$ & 0 & -1 & $\beta_{2}\left(\mathrm{x}_{2}\right)$ & 0 \\
\hline
\end{tabular}

Expressing the class of estimators $\mathrm{t}_{\mathrm{N}}$ at equation (2.1) in terms of $e$ 's, we have

$$
\mathrm{t}_{\mathrm{N}}=\overline{\mathrm{Y}}\left(1+\mathrm{e}_{0}\right)\left[\mathrm{w}_{1}\left(1+\mathrm{e}_{1}\right)^{\delta}\left\{1+\gamma_{1} \mathrm{e}_{1}+\frac{3}{2} \gamma_{1}^{2} \mathrm{e}_{1}^{2}\right\}+\mathrm{w}_{2}\left(1+\mathrm{e}_{2}\right)^{\beta}\left\{1+\gamma_{2} \mathrm{e}_{2}+\frac{3}{2} \gamma_{2}^{2} \mathrm{e}_{2}^{2}\right\}\right]
$$

where $\gamma_{1}=\frac{\eta_{1} \bar{X}_{1}}{2\left(\eta_{1} \bar{X}_{1}+\lambda_{1}\right)} \quad$ and $\quad \gamma_{2}=\frac{\eta_{2} \bar{X}_{2}}{2\left(\eta_{2} \bar{X}_{2}+\lambda_{2}\right)}$

Simplifying equation (2.3) and retaining terms to the first order of approximation, we have

$$
\begin{aligned}
& \left(\mathrm{t}_{\mathrm{N}}-\overline{\mathrm{Y}}\right)=\overline{\mathrm{Y}}\left[\mathrm{w}_{1}\left(1+\mathrm{e}_{0}-\mathrm{A}\left(\mathrm{e}_{1}+\mathrm{e}_{0} \mathrm{e}_{1}\right)+\mathrm{De}_{1}^{2}\right)+\mathrm{w}_{2}\left(1+\mathrm{e}_{0}-\mathrm{C}\left(\mathrm{e}_{2}+\mathrm{e}_{0} \mathrm{e}_{2}\right)+D \mathrm{e}_{2}^{2}\right)-1\right] \\
& \text { where, } \quad \mathrm{A}=\delta-\gamma_{1} \quad, \quad \mathrm{~B}=\frac{3}{2} \gamma_{1}^{2}-\delta \gamma_{1}+\frac{\delta(\delta-1)}{2} \\
& \mathrm{C}=\beta-\gamma_{2} \quad \text { and } \quad \mathrm{D}=\frac{3}{2} \gamma_{2}^{2}-\beta \gamma_{2}+\frac{\beta(\beta-1)}{2}
\end{aligned}
$$


Squaring both sides of equation (2.4) and taking expectations of both sides; we get the MSE of the estimator $t_{\mathrm{N}}$ to the first order of approximation, as

$$
\begin{aligned}
\operatorname{MSE}\left(\mathrm{t}_{\mathrm{N}}\right)= & \overline{\mathrm{Y}}^{2}\left[1+\mathrm{w}_{1}^{2} \mathrm{~A}_{1}+\mathrm{w}_{2}^{2} \mathrm{~A}_{2}-2 \mathrm{w}_{1} \mathrm{~A}_{3}-2 \mathrm{w}_{2} \mathrm{~A}_{4}+2 \mathrm{w}_{1} \mathrm{w}_{2} \mathrm{~A}_{5}\right] \\
\text { where, } \mathrm{A}_{1} & =\left\{1+\mathrm{f}_{1}\left(\mathrm{C}_{\mathrm{y}}^{2}+\mathrm{C}_{\mathrm{x}_{1}}^{2}\left(\mathrm{~A}^{2}+2 \mathrm{~B}\right)-4 \mathrm{~A} \rho_{\mathrm{yx}_{1}} \mathrm{C}_{\mathrm{y}} \mathrm{C}_{\mathrm{x}_{1}}\right)\right\} \\
\mathrm{A}_{2} & =\left\{1+\mathrm{f}_{1}\left(\mathrm{C}_{\mathrm{y}}^{2}+\mathrm{C}_{\mathrm{x}_{2}}^{2}\left(\mathrm{C}^{2}+2 \mathrm{D}\right)-4 \mathrm{C} \rho_{\mathrm{yx}_{2}} \mathrm{C}_{\mathrm{y}} \mathrm{C}_{\mathrm{x}_{1}}\right)\right\} \\
\mathrm{A}_{3} & =\left\{1-\mathrm{f}_{1} \mathrm{C}_{\mathrm{x}_{1}}\left(A \rho_{\mathrm{yx}_{1}} \mathrm{C}_{\mathrm{y}}-\mathrm{BC} \mathrm{x}_{1}\right)\right\} \\
\mathrm{A}_{4} & =\left\{1-\mathrm{f}_{1} \mathrm{C}_{\mathrm{x}_{2}}\left(C \rho_{\mathrm{yx}_{2}} \mathrm{C}_{\mathrm{y}}-\mathrm{DC} \mathrm{C}_{\mathrm{x}_{2}}\right)\right\} \\
\mathrm{A}_{5} & =\left\{1+\mathrm{f}_{1}\left(\mathrm{C}_{\mathrm{y}}^{2}+\mathrm{BC}_{\mathrm{x}_{1}}^{2}+\mathrm{DC}_{\mathrm{x}_{1}}^{2}-2 \mathrm{~A} \rho_{\mathrm{yx}_{1}} \mathrm{C}_{\mathrm{y}} \mathrm{C}_{\mathrm{x}_{1}}-2 \mathrm{C} \rho_{\mathrm{yx}_{2}} \mathrm{C}_{\mathrm{y}} \mathrm{C}_{\mathrm{x}_{2}}+A \mathrm{AC}_{\mathrm{x}_{1} \mathrm{x}_{2}} \mathrm{C}_{\mathrm{x}_{1}} \mathrm{C}_{\mathrm{x}_{2}}\right)\right\}
\end{aligned}
$$

The MSE of the class of estimator $t_{N}$ at equation (2.5) is minimised for the optimum values of $\mathrm{w}_{1}$ and $\mathrm{w}_{2}$ given as

$$
\mathrm{w}_{1}^{*}=\frac{\left(\mathrm{A}_{2} \mathrm{~A}_{3}-\mathrm{A}_{4} \mathrm{~A}_{5}\right)}{\left(\mathrm{A}_{1} \mathrm{~A}_{2}-\mathrm{A}_{5}^{2}\right)} \quad \text { and } \quad \mathrm{w}_{2}^{*}=\frac{\left(\mathrm{A}_{1} \mathrm{~A}_{4}-\mathrm{A}_{3} \mathrm{~A}_{5}\right)}{\left(\mathrm{A}_{1} \mathrm{~A}_{2}-\mathrm{A}_{5}^{2}\right)}
$$

The minimum MSE of estimator $t_{\mathrm{N}}$ is given by

$$
\operatorname{MSE}\left(\mathrm{t}_{\mathrm{N}}\right)=\overline{\mathrm{Y}}^{2}\left[1+\mathrm{w}_{1}^{* 2} \mathrm{~A}_{1}+\mathrm{w}_{2}^{* 2} \mathrm{~A}_{2}-2 \mathrm{w}_{1}^{*} \mathrm{~A}_{3}-2 \mathrm{w}_{2}^{*} \mathrm{~A}_{4}+2 \mathrm{w}_{1}^{*} \mathrm{w}_{2}^{*} \mathrm{~A}_{5}\right]
$$

\section{Empirical study}

Data Statistics: The data used for empirical study has been taken from the book "The Sampling Survey- Theory, Methods and Practice by Feng and Shi (1996) and the statistics calculated from raw data are given as follows:

Here we assume to take the sample size $\mathrm{n}=70$ from population $\mathrm{N}=180$ using SRSWOR.

$\mathrm{N}=180, \mathrm{n}=70, \overline{\mathrm{Y}}=13.9951, \overline{\mathrm{X}}_{1}=27.3981, \overline{\mathrm{X}}_{2}=38.7167, \mathrm{C}_{\mathrm{y}}=0.4180, \mathrm{C}_{\mathrm{x}_{1}}=0.4254$, $\mathrm{C}_{\mathrm{x}_{2}}=0.3339, \quad \rho_{\mathrm{yx}_{1}}=0.5630, \quad \rho_{\mathrm{yx}_{2}}=0.5273, \quad \rho_{\mathrm{x}_{1} \mathrm{x}_{2}}=0.2589, \quad \beta_{2}\left(\mathrm{x}_{1}\right)=4.2724$ $\beta_{2}\left(x_{2}\right)=2.1546$ 
The following table shows comparison between some existing estimators and proposed class estimators with respect to usual estimator.

Table 3.1: Variances / minimum MSEs and PRE's of different Estimators

\begin{tabular}{lcc}
\hline Estimators & MSE & PRE with respect to $\bar{y}$ \\
\hline $\mathrm{V}(\overline{\mathrm{y}})$ & 0.2988 & 100.00 \\
$\mathrm{MSE}_{\text {min }}\left(\mathrm{t}_{\mathrm{MR}}\right)$ & 0.1576 & 189.57 \\
$\mathrm{MSE}_{\text {min }}\left(\mathrm{t}_{\mathrm{r} 1}\right)$ & 0.1575 & 189.69 \\
$\mathrm{MSE}_{\text {min }}\left(\mathrm{t}_{\mathrm{r} 2}\right)$ & 0.1922 & 155.43 \\
$\mathrm{MSE}_{\text {min }}\left(\mathrm{t}_{\mathrm{reg}}\right)$ & 0.1669 & 179.01 \\
$\mathrm{MSE}_{\text {min }}\left(\mathrm{t}_{\mathrm{L}}\right)$ & 0.1574 & 189.81 \\
$\mathrm{MSE}_{\text {min }}\left(\mathrm{t}_{\mathrm{N} 1}\right)$ & 0.1669 & 179.01 \\
$\mathrm{MSE}_{\text {min }}\left(\mathrm{t}_{\mathrm{N} 2}\right)$ & 0.2041 & 146.38 \\
$\mathrm{MSE}_{\text {min }}\left(\mathrm{t}_{\mathrm{N} 3}\right)$ & 0.0868 & 344.20 \\
$\mathrm{MSE}_{\text {min }}\left(\mathrm{t}_{\mathrm{N} 4}\right)$ & 0.0815 & 366.58 \\
$\mathrm{MSE}_{\text {min }}\left(\mathrm{t}_{\mathrm{N} 5}\right)$ & 0.0867 & 344.67 \\
$\mathrm{MSE}_{\text {min }}\left(\mathrm{t}_{\mathrm{N} 6}\right)$ & 0.0812 & 367.93 \\
$\mathrm{MSE}_{\text {min }}\left(\mathrm{t}_{\mathrm{N} 7}\right)$ & 0.2000 & 149.38 \\
$\mathrm{MSE}_{\text {min }}\left(\mathrm{t}_{\mathrm{N} 8}\right)$ & 0.0808 & 369.37 \\
$\operatorname{MSE}_{\text {min }}\left(\mathrm{t}_{\mathrm{N} 9}\right)$ & $\mathbf{0 . 0 8 0 5}$ & $\mathbf{3 7 1 . 1 3}$ \\
${ }$ & &
\end{tabular}

Table 3.1 exhibits that the estimators based on auxiliary variables are more efficient than the one $(\bar{y})$ which does not utilize the auxiliary information. The members $t_{\mathrm{N} 3}, t_{\mathrm{N} 4}, t_{\mathrm{N} 5}$, $t_{N 6}, t_{N 8}$ and $t_{N 9}$ of the proposed class of estimators $t_{N}$ are equally efficient but all the estimators of proposed class are more efficient than the usual estimator, regression estimator using two auxiliary variable, traditional multivariate estimator using two auxiliary variable $\mathrm{t}_{\mathrm{MR}}$, estimator $\mathrm{t}_{\mathrm{r} 1}$ and $\mathrm{t}_{\mathrm{r} 2}$ (due to Abu-Dayeh et al. (2003)), and the estimator $\mathrm{t}_{\mathrm{L}}$, envisaged by Lu and Yan (2014).

\section{Conclusion}

In this present study we have suggested a class of estimators of the population mean of study variable $y$ when information is available on two auxiliary variables. In addition, some exponential families are also the member of the proposed class of estimators. We have obtained the properties of the proposed class of estimators up to the first order of approximation. Moreover, it was found that the proposed class of estimators are more efficient than the some traditional estimators and some recently proposed estimators considered here for the given condition. 


\section{Acknowledgement}

The authors are very indebted to the anonymous referees for their valuable suggestions leading to improvement of the quality of contents and presentation of the original manuscript.

\section{References}

1. Abu-Dayeh WA, Ahmed MS, Ahmed RA, Muttlak HA (2003). Some Estimators of a Finite Population Mean Using Auxiliary Information. Appl. Math Comput, 139, 287-298.

2. Feng, SY., Shi XQ. (1996). The Sampling Survey-Theory, Method and Practice, Shanghai: Shanghai Scientific and Technical Publishers.

3. Hartley, H.O. and Ross, A. (1954). Unbiased ratio estimators. Nature, 174, 270271.

4. Kadilar and Cingi (2005). A new estimator using two auxiliary variables. Applied Mathematics and Computation, 162, 901-908.

5. Khoshnevisan, M., Singh, R., Chauhan, P., Sawan, N., and Smarandache, F. (2007). A general family of estimators for estimating population mean using known value of some population parameter(s), Far East Journal of Theoretical Statistics, 22 181-191.

6. Koyuncu, N., and Ozel, G., (2013). Exponential estimators using characteristics of Poisson distribution: An application to earthquake data, 11th International Conference of Numerical Analysis and Applied Mathematics DOI: $10.1063 / 1.4825796$.

7. Lu J, Yan Z (2014). A Class of Ratio Estimators of a Finite Population Mean $\begin{array}{lllll}\text { Using Two Auxiliary } & \text { VNE }\end{array}$ e89538,doi:10.1371/journal.pone.0089538

8. Lu, Jingli, Yan, Zaizai, Xiuyun Peng (2014). A New Exponential Ratio-Type Estimator with Linear Combination of Two Auxiliary Variables, PLoS ONE, DOI: 10.1371/journal.pone.0116124.

9. Lu, Jingli (2013). The Chain Ratio Estimator and Regression Estimator with linear Combination of Two Auxiliary Variables. PLoS ONE, 8(11): e81085.

10. Perri, P. F. (2007). Improved ratio-cum-product type estimators. Statist. Trans, 8:51-69.

11. Quenouille, M. H. (1956). Notes on bias in estimation, Biometrika, 43, 353-360.

12. Sharma, P. and Singh, R. (2014). Improved Ratio Type Estimators Using Two Auxiliary Variables under Second Order Approximation. Mathematical Journal of Interdisciplinary Sciences, Vol.2 (2), 193-204.

13. Singh, R., Cauhan, P., Sawan, N., and Smarandache, F. (2007). Auxiliary information and a priori values in construction of improved estimators. Renaissance High Press. 
14. Singh R and Kumar M. (2012). Improved estimators of population mean using two auxiliary variables in stratified random sampling. Pak. Jour. of Stat and Oper. Res., 8(1): 65-72.

15. Singh, M. P. (1965). On the estimation of ratio and product of the population parameters. Sankhya B 27:231-328.

16. Singh, M. P. (1967). Ratio cum product method of estimation. Metrika 12:34-42.

17. Singh, R. and Kumar, M. (2011). A note on transformations on auxiliary variable in survey sampling. MASA, 6:1, 17-19.

18. Tailor, R., Chouhan, S. and Garg, N. (2012). A ratio-cum-product estimator of population mean in stratified random sampling using two auxiliary variables. STATISTICA, anno LXXII, n. 3.

19. Verma H.K., Sharma, P. and Singh, R. (2015). Some Families of Estimators Using Two auxiliary variables in stratified random sampling. Revista Investigacion Operacional, 36(2), 140-150. 